\title{
De la France vers la Saxe - Des galets peints du Mas d'Azil (Ariège, France) dans les collections archéologiques de la Saxe
}

From France to Saxony - Painted pebbles from Le Mas d'Azil (Ariège, France) in the archaeological collections from Saxony

Johann Friedrich Tolksdorf, Harald Floss et Ingo Kraft

\section{OpenEdition}

Journals

Édition électronique

URL : http://journals.openedition.org/paleo/3159

DOI : 10.4000/paleo.3159

ISSN : 2101-0420

Éditeur

SAMRA

Édition imprimée

Date de publication : 30 décembre 2016

Pagination : 297-305

ISSN : 1145-3370

Référence électronique

Johann Friedrich Tolksdorf, Harald Floss et Ingo Kraft, « De la France vers la Saxe - Des galets peints du Mas d'Azil (Ariège, France) dans les collections archéologiques de la Saxe », PALEO [En ligne], 27 | 2016, mis en ligne le 01 juin 2018, consulté le 07 juillet 2020. URL : http://journals.openedition.org/ paleo/3159 ; DOI : https://doi.org/10.4000/paleo.3159

\section{(c) $)(9)$}

PALEO est mis à disposition selon les termes de la licence Creative Commons Attribution - Pas d'Utilisation Commerciale - Pas de Modification 4.0 International. 


\title{
De la France vers la Saxe - Des galets peints du Mas d'Azil (Ariège, France) dans les collections archéologiques de la Saxe
}

\author{
Johann Friedrich TOLKSDORF(a) Harald FLOSS $^{(b)}$, \\ Ingo KRAFT(c)
}

Résumé : Cette contribution est consacrée à une trentaine de galets peints du Mas d'Azil qui ont été dénichés récemment dans le Staatliches Museum für Archäologie à Chemnitz (Allemagne). L'étude retrace l'historique muséographique de ces pièces et rappelle qu'elles ont été directement offertes, en 1899, par le fouilleur Édouard Piette à la collection royale préhistorique du musée de Dresde. L'identification de la provenance rend peu probable le fait qu'il s'agisse de contrefaçons et offre un complément important à l'inventaire de ces pièces emblématiques de la préhistoire française.

Mots-clés : galets peints, Le Mas d'Azil, Azilien, Édouard Piette, collections de Saxe.

Abstract: From France to Saxony - Painted pebbles from Le Mas d'Azil (Ariège, France) in the archaeological collections from Saxony. This contribution presents about 30 painted pebbles from Le Mas d'Azil which have been recently detected in the Staatliches Museum für Archäologie à Chemnitz (Germany). This study retraces the history of these pieces since their discovery at the site and demonstrates that they have been directly donated, in 1899, by the excavator Édouard Piette to the royal collection of prehistory at Dresden. The identification of their origin makes it unlikely that they represent forgeries and offer an important addendum to the existing inventory of these emblematic pieces of French prehistory.

Key-words: painted pebbles, Le Mas d'Azil, Azilian, Édouard Piette, collections of Saxony.

Les débuts de la recherche sur le Paléolithique en Saxe s'effectuent dans la sphère d'influence des travaux analogues en France. Au $19^{\circ}$ siècle, la découverte et l'étude systématique des dépôts karstiques a augmenté les interrogations sur les cultures paléolithiques, préoccupation partagée aussi bien par le milieu de la recherche, que par le grand public. Ainsi, l'œuvre de Charles Darwin « On the origin of species » initia une discussion autour de la dimension chronologique et qualitative de la création humaine, jusqu'alors peu développée. Les sites français constituaient à cette époque un centre d'intérêt principal ; c'est la raison pour laquelle l'attention des collectionneurs s'est tournée dans cette direction (Kraft 2010). Cet intérêt a déclenché un vigoureux commerce de vestiges paléolithiques. Par la suite, beaucoup de ces collections privées ont constitué la base de collections publiques. La collection préhistorique de Dresde, qui a été inaugurée en 1874 comme une partie du Musée royal de minéralogie et

(a) Landesamt für Archäologie Sachsen, Projekt ArchaeoMontan, Zur Wetterwarte 7, D 01109 Dresden,

JohannFriedrich.Tolksdorf@lfa.sachsen.de

(b) Eberhard Karls Universität Tübingen, Abteilung Ältere Urgeschichte und Quartärökologie, D 72070 Tübingen,

harald.floss@uni-tuebingen.de

(c) Landesamt für Archäologie Sachsen, Referatsleiter Ostsachsen, Zur Wetterwarte 7, D 01109 Dresden, ingo.kraft@Ifa.sachsen.de 
géologie, et qui exposait à partir de 1892 dans le palais du Zwinger des vestiges et des répliques provenant de sites français, est un exemple typique de cette époque (Schachtmann 2010). La collection en tant que telle doit son existence à des influences venues de France, étant donné que le premier conservateur de la collection, Hanns Bruno Geinitz (1814-1900), était parmi les visiteurs admiratifs de l'exposition universelle de Paris en 1867 et notamment des vestiges paléolithiques récemment découverts (Coblenz 2000).

Ce musée doit ses acquisitions de collections, et cela de manière considérable, à la société des sciences naturelles "ISIS », fondée en 1833, et qui, à partir de 1870, consacrait une série de conférences à la préhistoire (Coblenz 1993). Au sein de cette société, se sont rencontrés les principaux représentants de la jeune recherche archéologique de la Saxe, lesquels disposaient d'une bonne interconnexion avec les figures centrales de la recherche préhistorique française. À titre d'exemple, ces relations personnelles se sont constituées, entre autres, à partir d'Ida von Boxberg, la première archéologue saxonne (Mélard et Krabath 2005 ; Herrmann et Krabath 2013). Elle était personnellement connue des protagonistes de la recherche paléolithique française comme participante aux fouilles en France où elle a acquis, au cours de ses séjours d'études, de nombreux vestiges parvenus, après sa mort, dans les collections de Dresde. Une conférence de préhistoire de la société ISIS, datée de 1882, illustre bien la façon dont le regard porté sur la France était important pour la recherche paléolithique en cours de formation à ce moment-là en Saxe. Hanns Bruno Geinitz a donné une conférence intitulée «Sur l'état actuel de la recherche préhistorique en France et en Allemagne ", dans laquelle il fait état des progrès récents de la recherche française et essaye de comparer celle-ci à celle de l'Allemagne dans le but d'en déduire un programme d'activités pour la recherche préhistorique de ce pays (Geinitz 1882).

La présence de vestiges paléolithiques originaires de France (par exemple de Saint- Acheul, Abbeville, Grotte Margot) est également bien perceptible à travers le catalogue raisonné de la collection de Dresde, établi par Johannes Deichmüller (1854-1944). Cet intense commerce de pièces archéologiques à l'échelle européenne, est également à l'origine de l'arrivée, à Dresde, d'un lot de pièces remarquables provenant de la grotte du Mas d'Azil. Dans le supplément au catalogue intitulé « Enrichissement de la collection préhistorique 1889 à 1930 » on trouve pour l'année 1899, la mention de " 31 galets peints de la grotte du Mas d'Azil, Dépt. Ariège, France » (fig. 1B). Comme provenance est indiquée la mention «E. Piette de Rumigny " attestant de cette manière une attribution assurée à l'un des ensembles les plus emblématiques, mais aussi problématiques en termes d'authenticité : celui des galets peints du Mas d'Azil. L'ultime certitude sur la provenance est donnée par les comptes rendus des séances de la société ISIS dans lesquels on lit, pour le 18 janvier 1900 : «Prof. Dr. J. Deichmüller présente et décrit un certain nombre de galets peints de la grotte du Mas d'Azil dans les Pyrénées, qui ont été offerts à la collection royale préhistorique de Dresde par Monsieur Ed. PietteRumigny ».

Les échanges intenses du $19^{\circ}$ siècle avec la recherche paléolithique française font contraste avec la situation du $20^{\circ}$ siècle, qui est plutôt marquée par une concentration des travaux sur les sites saxons et une alimentation de la collection par des vestiges exclusivement autochtones. Cette réorientation est influencée, entre autres, par des modifications politiques et idéologiques et par les rivalités entre l'empire allemand de 1871 et la France, culminant dans les deux guerres mondiales et déclenchant aussi une rupture de nombreux contacts scientifiques. Un contrepoint anecdotique à cette évolution générale est donné, de 1940 à 1943, par l'étude de sites préhistoriques par des officiers français prisonniers de guerre, dans le cadre de « l'Oflag IV $D$ Elsterhorst (Nardt) " près de Hoyerswerda (Saxe) (Groupe d'Études Préhistoriques d'Elsterhorst 1950).

Après 1945, les relations des préhistoriens de la République fédérale allemande (RFA) avec la recherche préhistorique française se sont développées de manière plus satisfaisante mais, pour les collègues travaillant dans les institutions de l'Allemagne de l'Est, les échanges scientifiques avec la France étaient particulièrement difficiles (Gramsch 2010). Dans ce contexte politique, les collections issues des parties du Reich allemand, occupées par I'Union Soviétique, se sont également trouvées éloignées de la recherche internationale. Cette « amnésie » a été également lourde de conséquences pour les vestiges de la collection de Dresde, fortement détruite pendant la Seconde Guerre mondiale, ainsi que les vestiges du Mas d'Azil le démontrent clairement. Quand Claude Couraud entreprend, en 1985, sa révision des galets peints, son catalogue du site du Mas d'Azil comprend presque uniquement des collections de France et d'Angleterre (Couraud 1985). Des addenda méticuleux par Paul Bahn (Bahn, Cole et Couraud 1987) ont permis de dénicher quelques galets peints supplémentaires, issus de collections des États-Unis, mais le lot de Dresde est resté totalement négligé, ce qui montre de quelle manière dramatique les collections anciennes du centre et de l'Est de l'Allemagne sont restées éloignées de la recherche paléolithique française. Le but de cet article est donc de donner une présentation des galets peints du Mas d'Azil issus de la collection de Dresde, qui restent jusqu'à aujourd'hui quasiment inconnus en France. Pour comprendre l'ampleur de l'éparpillement des galets décorés de ce site et les complications liées à l'authenticité de certaines collections suite à des falsifications, il convient de retracer d'abord de manière succincte l'historique compliqué de la recherche du Mas d'Azil.

\section{Le site}

Le site archéologique ariègeois du Mas d'Azil, grande grotte traversée par l'Arise, est essentiellement localisé sur les terrasses situées sur les deux rives de la rivière. Suite à des découvertes d'ossements dans le cadre d'une construction de route, Édouard Piette (1827-1906) y entreprend des fouilles à partir de 1887 et vraisemblablement jusqu'en 1889 

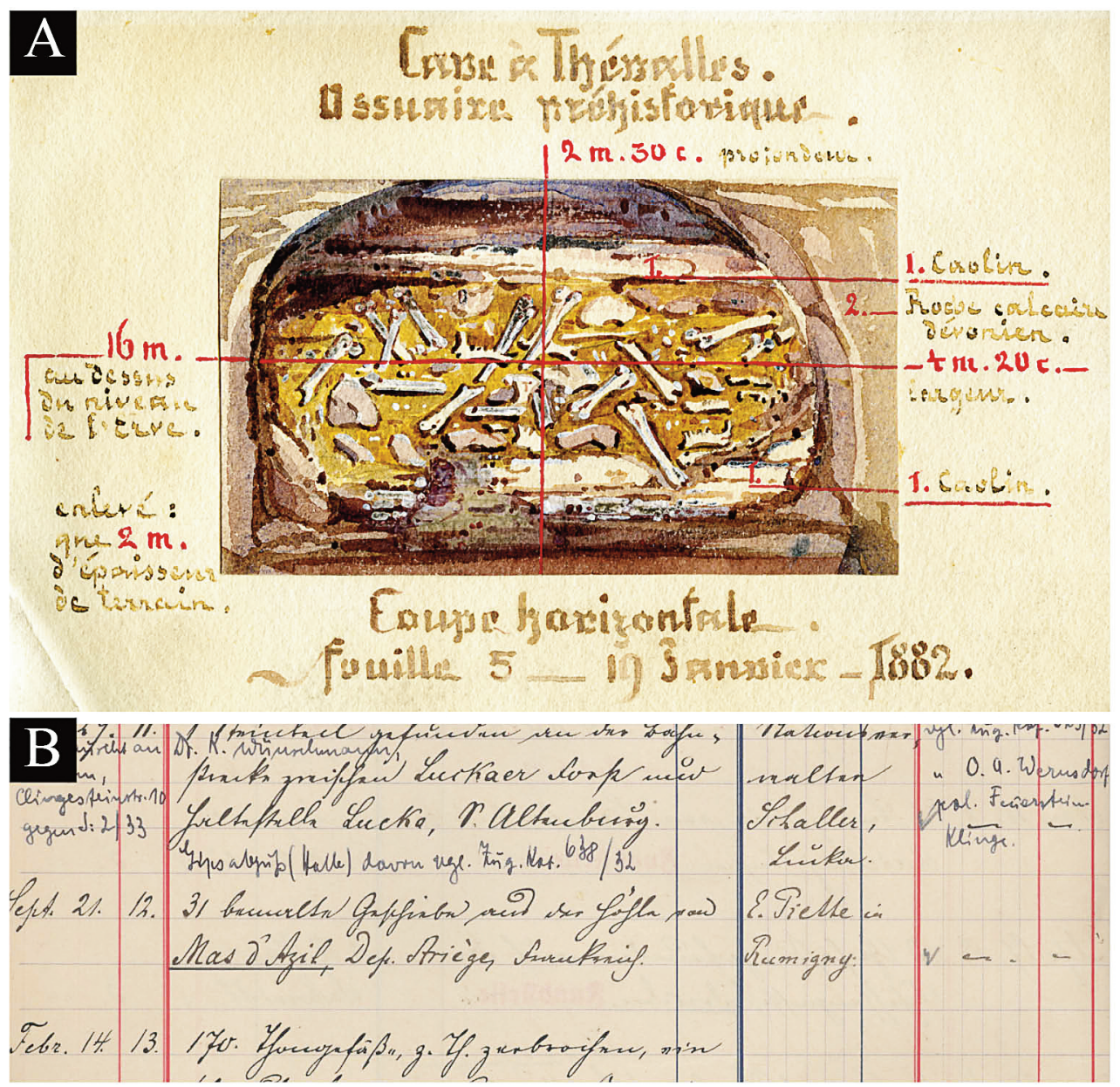

Figure 1 A - Aquarelle d'Ida von Boxberg de 1885, illustrant la stratigraphie de la Grotte de Thévalles. $B$ : extrait du catalogue des entrées de la collection préhistorique royale de Dresde, témoignant de l'entrée de 31 galets peints du Mas d'Azil, le 21 septembre 1899, et indiquant E. Piette comme donateur de ces pièces.

Figure 1 A - Watercolor by Ida von Boxberg from 1885, showing a stratigraphy of the Grotte de Thévalles. $B$ : extract of the catalogue of the royal prehistoric collection of Dresden, indicating the entry of 31 painted pebbles from Le Mas d'Azil, dated to the 21st of September 1899, and indicating E. Piette as donator of these pieces.

(Kegler 2007). Habitué à la notion de la stratigraphie et des fossiles-directeurs, Edouard Piette distingue, entre trois niveaux de Magdalénien, séparés par des dépôts de crue, et le Néolithique, un niveau épais dit « de transition ». Pour lui, cette série de dépôts représentait le comblement correspondant à la lacune existante, pour l'époque, entre les cultures paléolithiques et le Néolithique. Ce niveau était caractérisé par une faune et une flore de climat tempéré. Dans le cadre d'une subdivision fine, il distinguait, pour ce niveau de transition, une phase stratigraphique plus récente, se distinguant par un taux élevé de coquilles de mollusques (couche à escargots) et une phase plus ancienne, comportant quant à elle les galets peints (couche à galets coloriés). Pour cette phase entre Magdalénien et Néolithique, Edouard Piette introduit finalement le terme «Azilien » pour lequel les galets peints constituent un critère de définition (Piette 1895, Kegler 2007). Dans une autre publication (Piette 1896), l'auteur se consacre uniquement à ces pièces et leur attribue une signification de communication, similaire à l'alphabet.

D'un point de vue archéologique, la suite des recherches dans la cavité est moins réjouissante (Kegler 2017). Certes, le site a conquis l'intérêt du public et a attiré des personnalités scientifiques, comme par exemple Henri Breuil (fouilles 1901-1902), mais au début du $20^{\circ}$ siècle, le site était progressivement laissé à l'abandon et affecté par de nombreuses activités clandestines et désordonnées. Dans la revue Nature, une contribution de 1926 décrit l'état complètement insupportable du site : "Practically there is no control, no protection and no organised excavation whatever at Mas d'Azil. There are masses of valuable material, but none of it is being worked at properly. Much of it, I fear, is being wasted and muddled up. There is a 
"guide", a pleasant untrained man, who pokes about in the caves, digs out bones which, as he remarks, fall to pieces, and presents the casual visitor with teeth or flint implements he has found in his own researches. He has no regular salary. He has to supplement his fees and tips by other work. Occasionally, isolated individuals obtain permission from the municipality and prod in the rocks and extract this or that and publish their "results", according to their lights. There is a small useless museum without labels or arrangement at the Mairie." (Well 1926). On doit attendre jusqu'en 1935, pour que de nouvelles fouilles soient effectuées par Saint-Just et Marthe Péquart, qui les prolongent jusqu'en 1944. La documentation de ces travaux demeure cependant très lacunaire (Kegler 2007). Après la Seconde Guerre Mondiale, des travaux de sécurisation des dépôts ont été réalisés, sous la direction d'André Alteirac ; ils ont donné lieu à d'autres fouilles, malheureusement inédites. En résumé, l'historique difficile de la recherche, et l'éparpillement des vestiges vers d'innombrables collections, ont malheureusement rendus compliquée une étude complète de ce site (Kegler 2007).

\section{Des galets peints - objets recherchés par les collectionneurs et les faussaires}

Malgré ces problèmes, le site du Mas d'Azil a connu une réputation non négligeable comme site de transition, attisant l'intérêt de nombreux musées et collectionneurs désireux de posséder des pièces provenant de ce site, convoitise qui a déclenché une intense activité d'échanges et d'acquisitions. Les galets peints sont au premier plan de l'intérêt pour ce site, eux qui se trouvaient, depuis leur découverte, au centre d'interprétations fantaisistes. J. Deichmüller cite par exemple, pour les pièces de Dresde, dans le cadre de son exposé en 1900 devant la société ISIS, les interprétations d'E. Piette, d'après lesquelles ces objets représentaient des images du soleil, des illustrations d'arbres, et de systèmes très anciens de comptage et d'écriture. Aujourd'hui, le spectre des interprétations s'étend d'un proto-système d'écriture (Piette 1896 ; 1904), d'une utilisation comme objets de culte similaire aux «Churingas » australiens (Cook 1903), de pions de jeu, de l'art pour l'art (Mallery 1893 ; Péquart et Péquart 1936) jusqu'à une représentation cyclique du genre calendrier (Bahn et Couraud 1984, Couraud 1985). La nature énigmatique de ces galets en a fait des objets idéaux pour les collections et expositions de la charnière des $19^{\circ}$ et $20^{\circ}$ siècles, qui cherchaient toujours à présenter de nouvelles catégories d'objets surprenants. Ces circonstances ont contribué à la distribution confuse et non-documentée d'une grande partie de ces vestiges. Les travaux de Claude Couraud (1985) ont révélé l'ampleur de l'éparpillement de ces pièces, réunissant 1400 pièces issues d'une vingtaine de musées, surtout français et anglais. Cette liste a été enrichie ultérieurement par la présentation de vestiges supplémentaires provenant de France (Couraud et Alteirac 1990, 1993 ; Alteirac et Couraud 1996) (fig. 2A, 2B). Quelques pièces ont même été dénichées aux États-Unis (Bahn et al. 1987) et au Zimbabwe. Mais il n'était pas possible, à ce moment-là, de prendre en considération les collections de l'ex-bloc des pays de l'Est. Les pièces achetées par le musée de pré- et protohistoire de Berlin en 1929 (Unverzagt et von Jenny 1935, 4) et les pièces de Dresde lui ont donc échappé. Le nombre comptabilisé de 1619 galets peints du Mas d'Azil (Alteirac et Couraud 1996) ne représente donc qu'une quantité minimum.

Un résultat majeur de l'étude de Claude Couraud réside dans l'observation d'un taux élevé, mais variable suivant les collections, de pièces falsifiées (fig. 4). Les critères d'identification de ces contrefaçons modernes sont l'utilisation de pigments non-connus sur le site, des représentations stylistiquement peu cohérentes, ainsi que l'utilisation de surfaces fragmentées secondaires comme supports pour la peinture. D'autres galets se sont avérés authentiques, avec certitude, car couverts par une croûte calcaire post-dépositionnelle. Mais entre ces deux catégories, il y a un nombre élévé de cas ambigus, dont l'attribution tend seulement vers le vrai ou le faux. En ce qui concerne le choix et la composition de signes, Claude Couraud a pu mettre en évidence statistiquement que les compositions ne sont pas aléatoires, bien qu'il n'observe pas une combinaison de la totalité des croisements théoriquement possibles. II semble aussi que certaines quantités de signes prédominent (par exemple signes 21 ou 29).

En synthétisant les résultats de la totalité des collections étudiées par Claude Couraud (fig. 3), on remarque un taux considérable de pièces fausses issues des séries Piette. Malgré une certaine tendance selon laquelle la plupart des falsifications sont issues des phases de popularité croissante du site, on ne peut pas nier que les premières contrefaçons ont été réalisées dès les fouilles Piette. La coutume de payer les ouvriers pour la découverte de pièces spectaculaires a certainement entraîné la réalisation de fausses pièces.

\section{Les galets peints de la collection de Dresde}

Aujourd'hui, les collections archéologiques de la Saxe possèdent 32 galets peints du Mas d'Azil, dont 31 de la collection de 1899. L'inventaire établi après la Seconde Guerre Mondiale comptait 31 pièces, réparties en deux lots (28 plus 3). En 1959, deux de ces galets (fig. 4, numéros d'inventaire ID 000277348 et 00301207 ) ont été prêtés à la collection d'études de l'université Humboldt à Berlin, d'où ils ne sont rentrés à Dresde qu'en 2010. Miraculeusement, l'inventaire de 1980 répertorie une $32^{\circ}$ pièce (fig. 4, numéro ID 00089758). Malgré un manque d'informations concernant la provenance de cette pièce, la numérotation interne plaide en faveur d'une origine dans les collections Hauser, Wilke et Staffel. Ceci témoigne en faveur d'une acquisition après 1925. II est probable que cette pièce ait été vendue au musée par l'antiquaire suisse allemand Otto Hauser, personnage controversé, connu pour le trafic systématique de vestiges préhistoriques français, et également personnage illustrant de façon exemplaire la rupture entre l'archéologie française et allemande déclenchée par la Première Guerre Mondiale (Drößler et 

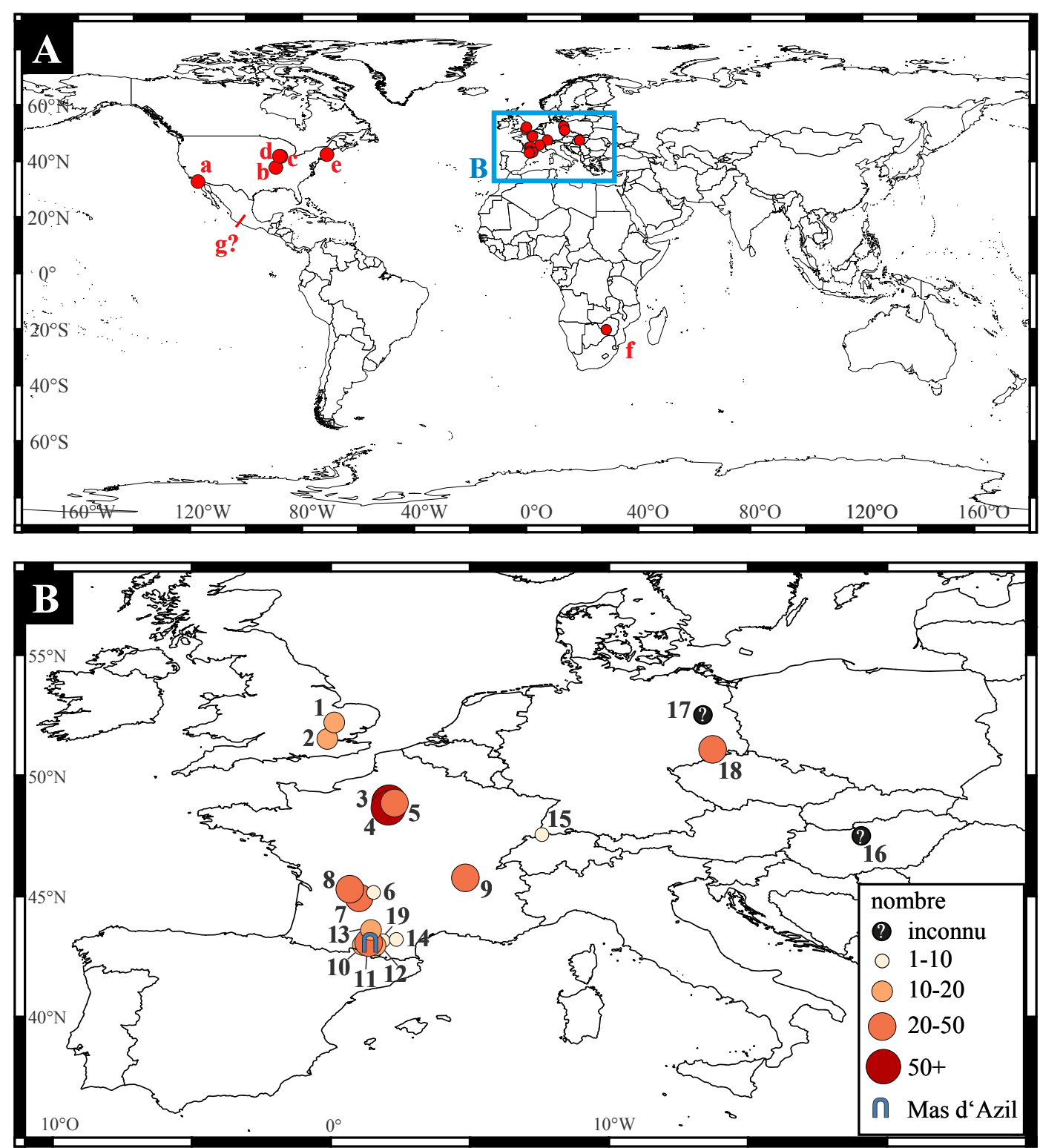

Figure 2 - A - Collections extra-européennes contenant des galets peints du Mas d'Azil, d'après Bahn et al. 1987 : a Museum de San Diego ; b Logan Museum d'Anthropologie à Beloit ; Murphysboro (White 1992) ; c Field Museum de Chicago/ université de Chicago ; $d$ Saint Procopius College, Lisle ; e Peabody Museum d'Archéologie et d'Ethnologie à Harvard, Cambridge ; $f$ National Museum de Rhodesia (aujourd'hui Musée National de Zimbabwe); g prêt Mexique. B - Collections de galets peints du Mas d'Azil en Europe suivant Couraud (1985) : 1 : Museum of Archaeology and Anthropology, Cambridge ; 2 : British Museum, London ; 3 : Musée d'Archéologie nationale, Saint-Germain-en-Laye ; 4 : Musée de l'Homme, Paris ; 5 : Institut de Paléontologie humaine, Paris; $6:$ Musée E. Rupin, Brive ; 7 : Musée national de Préhistoire, Les Eyzies-de-Tayac; 8 : Musée d'art et d'archéologie du Périgord, Périgueux; $9:$ Musée d'histoire naturelle - Guimet, Lyon ; 10 : Domaine de Pujol, Saint-Girons ; 11 : Musée de la Préhistoire, Le Mas-d'Azil ; 12 : Musée départemental de l'Ariège, Foix ; 13 : Musée d'Histoire naturelle de Toulouse, Toulouse ; 14 : Musée des Beaux-arts, Carcassonne ; 15 : Museum der Kulturen, Basel ; 16 : Magyar Nemzeti Museum, Budapest ; 17 : Museum für Vor- und Frühgeschichte, Berlin (Unverzagt/von Jenny 1935) ; 18 : Königliche Prähistorische Sammlung/AAS, Dresden ; 19 : Sammlung Pouech, Pamiers (Alteirac/Couraud 1996).

Figure 2 - A - Extra european collections containing painted pebbles from Le Mas d'Azil, from Bahn et al. 1987: a Museum of San Diego; $b$ Logan Museum of Anthropology at Beloit; Murphysboro (White 1992); c Field Museum of Chicago/ university of Chicago; $d$ Saint Procopius College, Lisle; e Peabody Museum of Archaeology and Ethnology at Harvard, Cambridge; $f$ National Museum of Rhodesia (today National Museum of Zimbabwe); $g$ loan Mexico. B - Collections of painted pebbles of Le Mas d'Azil in Europe following to Couraud (1985): 1: Museum of Archaeology and Anthropology, Cambridge; 2: British Museum, London; 3: Musée d'Archéologie nationale, Saint-Germain-enLaye; 4: Musée de l'Homme, Paris ; 5: Institut de Paléontologie humaine, Paris; 6: Musée E. Rupin, Brive; 7: Musée national de Préhistoire, Les Eyzies-de-Tayac; 8: Musée d'art et d'archéologie du Périgord, Périgueux; 9: Musée d'histoire naturelle - Guimet, Lyon; 10: Domaine de Pujol, Saint-Girons; 11: Musée de la Préhistoire, Le Mas-d'Azil; 12: Musée départemental de l'Ariège, Foix; 13: Musée d'Histoire naturelle de Toulouse, Toulouse; 14: Musée des Beaux-arts, Carcassonne; 15 Museum der Kulturen, Basel; 16: Magyar Nemzeti Museum, Budapest; 17: Museum für Vor- und Frühgeschichte, Berlin (Unverzagt/von Jenny 1935); 18: Königliche Prähistorische Sammlung/AAS, Dresden; 19: Sammlung Pouech, Pamiers (Alteirac/Couraud 1996). 


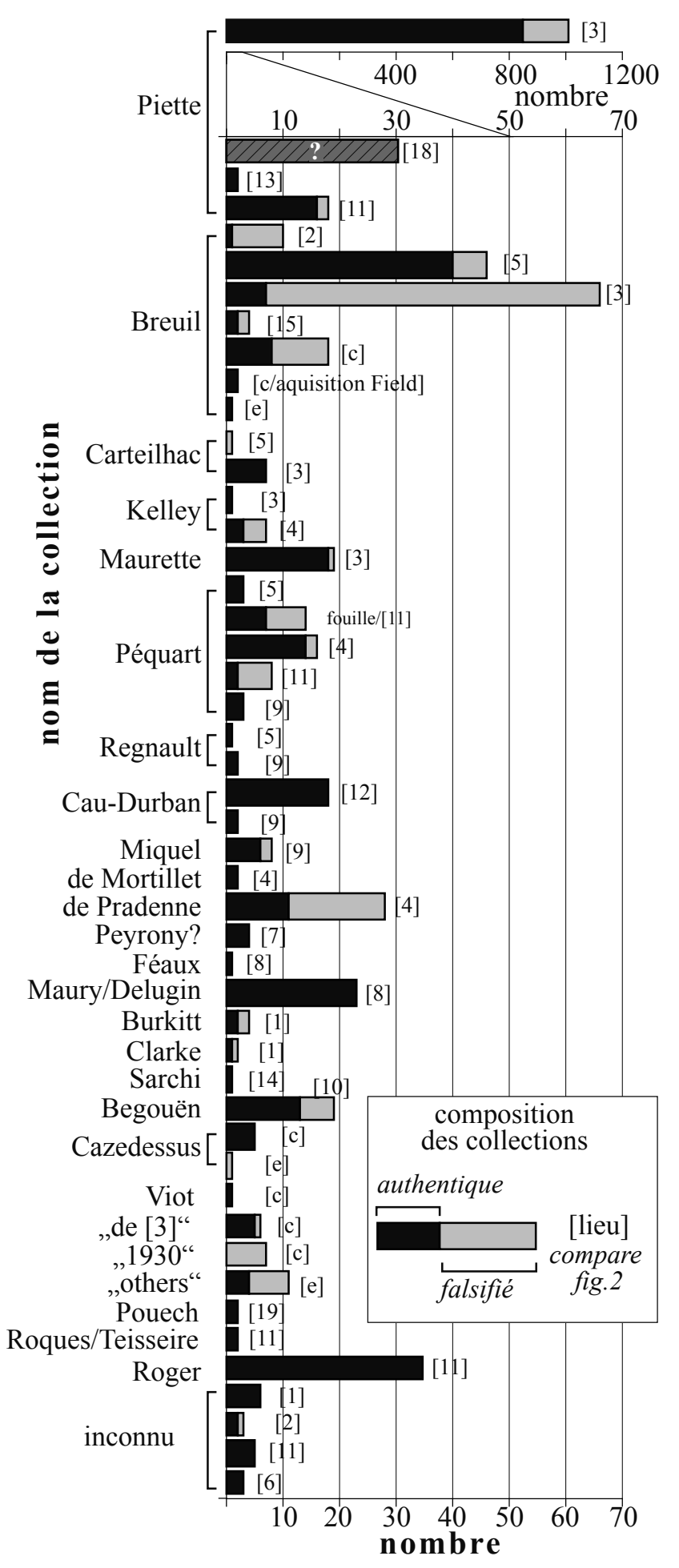

Figure 3 - Pourcentage des falsifications sur la base d'estimations de C. Couraud (Couraud 1985; Bahn et al. 1987; Couraud/Alteirac 1990; Alteirac/Couraud 1993; 1996). Les chiffres se réfèrent aux numéros des collections (voir fig. 2).

Figure 3 - Percentage of forgeries following the estimations by C. Couraud (Couraud 1985; Bahn et al. 1987; Couraud/ Alteirac 1990; Alteirac/Couraud 1993; 1996). The counts refer to the numbers of the collections (see fig. 2).
Freyberg 2001). La pièce en question montre, contrairement aux autres, l'étalement de la couleur sur une croûte de concrétions. Malgré le motif, peu suspect, de simples bandes rouges, cette observation plaide en faveur d'une contrefaçon. Cette pièce a été donc exclue du corpus des galets peints de 1899 .

Avec 31 exemplaires, la série de Dresde constitue un des plus importants ensembles de galets peints du Mas d'Azil dans le monde (fig. 3). De plus, elle fait partie des rares séries directement attribuables aux travaux archéologiques de l'inventeur Edouard Piette. Les motifs choisis représentent des bandes parallèles, des alignements de points, de croix, des lignes droites dans l'axe longitudinal de la pièce, ainsi que des empreintes digitales latérales. Ces motifs s'intègrent parfaitement dans le langage de formes connu pour ce site. D'un point de vue technologique et stylistique, aucun argument contre l'authenticité de ces pièces ne peut donc être émis.

Grâce à l'enquête de C. Couraud (1985) qui a pu détailler les taux divergents des falsifications dans les diverses collections, on arrive aujourd'hui à reconstituer plusieurs voies par lesquelles ces contrefaçons ont été introduites. Les enregistrements d'Edouard Piette nous apprennent que les ouvriers de la fouille, dans le contexte spécifique de cette époque, touchaient des primes pour la découverte de vestiges particuliers (Kegler 2007- p. 21). Ce type de procédé a pu inciter les ouvriers à falsifier des objets directement lors de la fouille. Ce type de comportement a pu être observé également sur d'autres fouilles de l'époque, comme par exemple au Kesslerloch près de Thayingen (Lindenschmit 1876). Toutefois, la variation du nombre des falsifications dans les différentes collections plaide en faveur de l'hypothèse, selon laquelle l'origine de ces faux ne se trouve pas forcément à la source, c'est-à-dire lors de la fouille, mais a pu intervenir ultérieurement dans le cadre du processus complexe de mise en place et d'acquisition des collections. En ce qui concerne la collection de Dresde, l'origine directe chez E. Piette, ainsi que les critères établis par C. Couraud n'offrent aucun indice de la présence de faux. La couche de pigments est pâteuse et régulière, et ne couvre jamais des éclatements plus récents des galets. Des restes d'un dépôt limoneux, ainsi que des microcharbons de bois, s'observent dans des fissures, ce qui coïncide avec les descriptions du niveau archéologique en question (Kegler 2007 - p. 57 et suiv.).

L'étude systématique de cette série de galets peints est loin d'être achevée. À côté de l'intérêt scientifique indubitable, ces galets du Mas d'Azil illustrent bien de quelle manière la recherche préhistorique allemande a été fascinée par la richesse des sites français. Le but principal de cette contribution est de faire connaître l'existence des objets de Dresde quasiment inconnus dans leur pays d'origine. En 2004, les pièces ont été intégrées dans l'exposition permanente du Staatliches Museum für Archäologie Chemnitz (SMAC) (fig. 5), et notamment dans une vitrine consacrée à l'historique de la recherche archéologique de la Saxe. On y a choisi la mise en scène d'une vieille valise, symbole de l'enthousiasme d'Ida von Boxberg, et de ses voyages parmi les sites préhistoriques de France. 


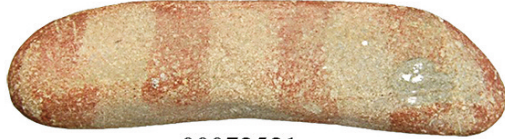

00072521

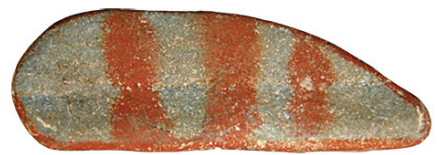

00095155
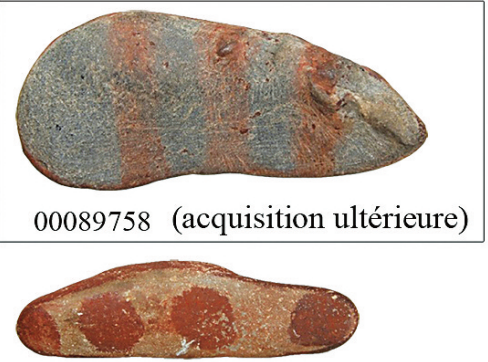

00072526

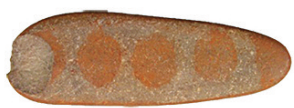

00095160

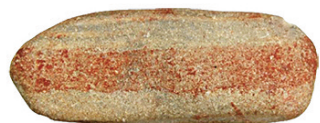

00072527

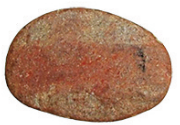

00095167

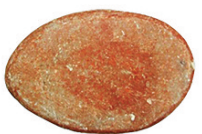

00072531

$3 \mathrm{~cm}$

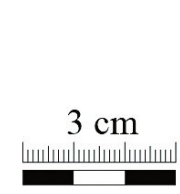

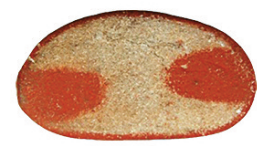

00072530

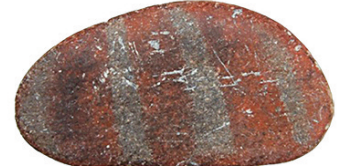

00089757

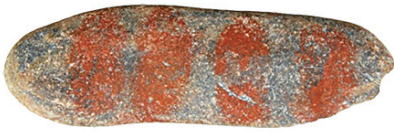

00095163

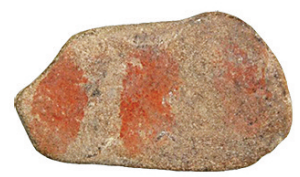

00095166

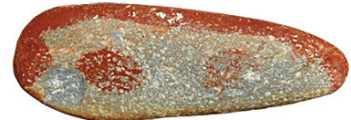

00095161

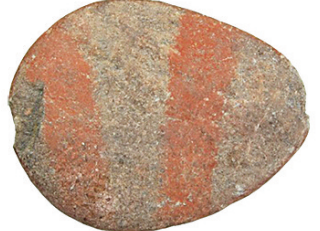

00089755

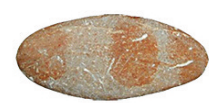

00072532

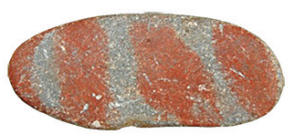

00089756

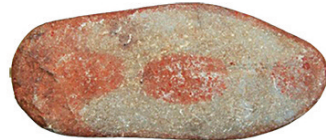

00095162

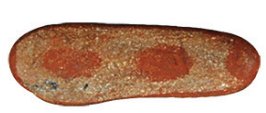

00095156

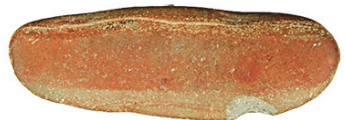

000277348

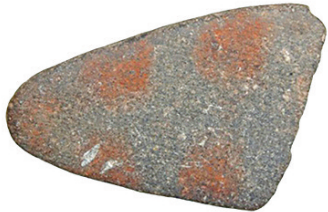

00072524

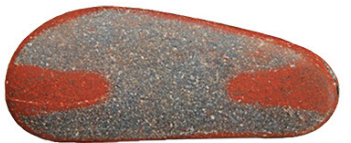

00072522

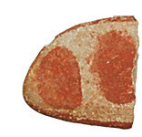

00072533

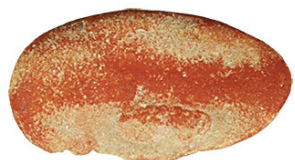

00072523

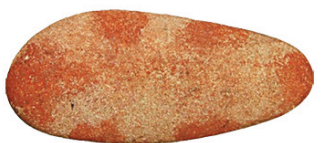

00072529

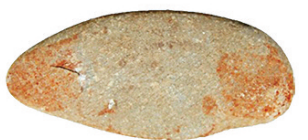

00072528

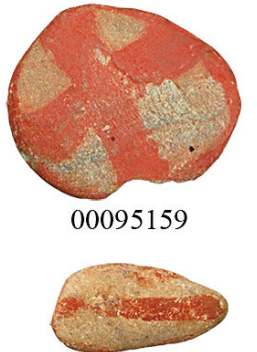

00095156

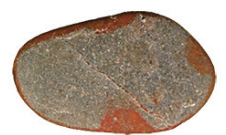

00095157

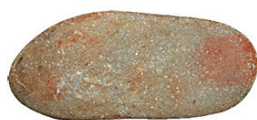

00072525

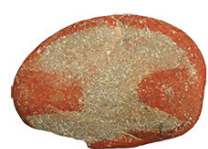

00095158

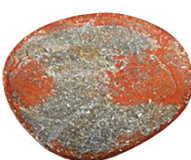

00301207

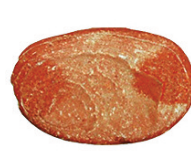

00095164

Figure 4 - Les galets peints du Mas d'Azil issus de l'archive archéologique de la Saxe avec leurs numéros d'inventaire et regroupés suivant les différents motifs. La pièce 00089758 ne fait très probablement pas partie de la collection de 1889 (E. Piette), mais est originaire d'une entrée plus récente, sans provenance fiable.

Figure 4 - The painted pebbles of Le Mas d'Azil from the archaeological archive of Saxony with their inventory numbers and grouped following their different themes. The piece 00089758 probably doesn't belong to the collection from 1889 (E. Piette) but belongs to a more recent museum entry, without reliable origin. 


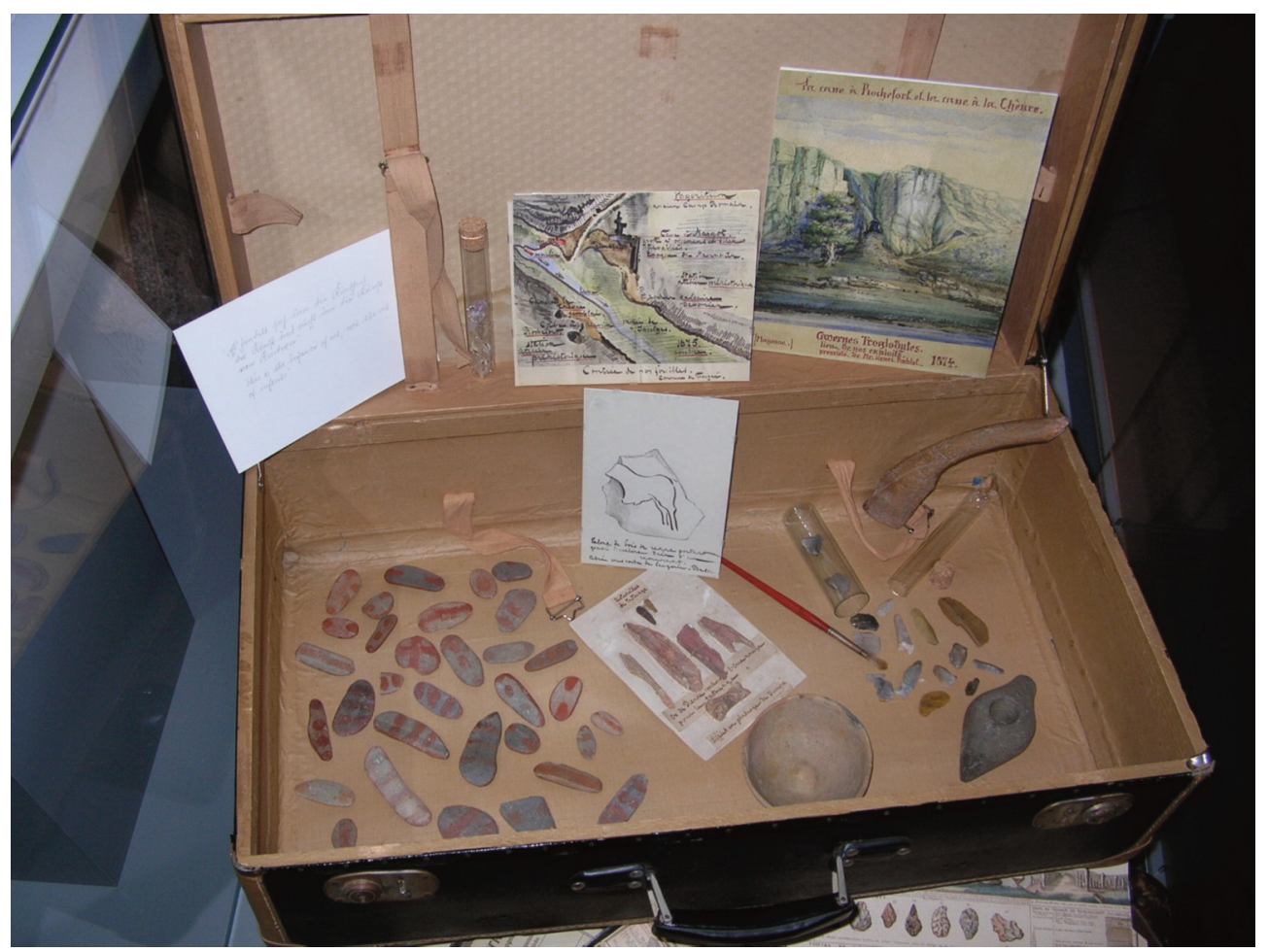

Figure 5 - Présentation actuelle des galets peints du Mas d'Azil au Staatliches Museum für Archäologie Chemnitz.

Figure 5 - The current presentation of the painted pebbles from Le Mas d'Azil in the Staatliches Museum für Archäologie Chemnitz.

\section{Références bibliographiques}

ALTEIRAC A., COURAUD C. 1996 - Des galets peints dans la collection Pouech. Bulletin de la Société préhistorique de l'Ariège 51, 1996, 129-132.

BAHN P.G. 1984 - Bahn How to spot a fake azilian pebble. Nature 308, 1984, 229.

BAHN P.G., COLE G.H., COURAUD C. 1987 - Les galets peints du Mas-d'Azil déposés dans les musées des EtatsUnis. Préhistoire Ariégeoise 42, 1987, 119-153.

BAHN P.G., COURAUD C. 1984 - Azilian pebbles: an unsolved mystery. Endeavour 8, 1984, 156-158.

COBLENZ W. 1993 - Die prähistorische Sektion der ISIS. Naturwissenschaftliche Gesellschaft Isis: Sitzungsberichte und Abhandlungen der Naturwissenschaftlichen Gesellschaft Isis, Dresden e.V. 1991/92, 1993, 35-38.

COBLENZ W. 2000 - Deichmüller als Senior der prähistorischen Forschung Sachsens. Naturwissenschaftliche Gesellschaft Isis: Sitzungsberichte und Abhandlungen der Naturwissenschaftlichen Gesellschaft Isis, Dresden e.V. 1995/96, 2000, 171-178.

COURAUD 1985 - L'art azilien: Origine-survivance. XX ${ }^{\ominus}$ supplément à Gallia Préhistorique, Editions du CNRS, Paris175 p.

COURAUD C., ALTEIRAC A. 1990 - Les galets peints de la collection Roger. Préhistoire Ariégeoise 45, 1990, 187-199.
COURAUD C., ALTEIRAC A. 1993 - Nouveaux galets peints du Mas-d'Azil. Bulletin de la Société préhistorique de l'Ariège 48, 1993, 31-33.

COOK A. 1903 - Les galets peints du Mas d'Azil. L'Anthropologie 14, 1903, 655-660.

DRÖSSLER R., FREYBERG M. 2001 - Der Schweizer Archäologe Otto Hauser und die „Wissenschaftliche Privatsammlung Otto Hauser" in Zeitz. Archäologie in Sachsen-Anhalt 1, 2002, 46-50.

GEINITZ H. 1882 - Über den gegenwärtigen Stand der prähistorischen Forschung in Frankreich und Deutschland. Sitzungsberichte und Abhandlungen der Naturwissenschaftlichen Gesellschaft Isis, 1882, 127-134.

GRAMSCH B. 2010 - Paläolithforschung in der ehemaligen DDR. Mitteilungen der Gesellschaft für Urgeschichte 19, 2010, 157-172.

GRIFFIN J.B., MELTZER D.J., SMITH B.D., STURTEVANT W.C. 1988 - A Mammoth Fraud in Science. American Antiquity 53, 1988, 578-582.

GROUPE d'Études Préhistoriques d'Elsterhorst 1950 - Le gisement d'Elsterhorst (Haute-Lusace) et la civilisation à caractère mésolithique de l'Elster et de la Sprée. Compte rendu de la XIII session du Congrès Préhistorique de France, Paris, 1950 (Paris 1950) 328-353.

HERRMANN E., KRABATH S., von BOXBERG I. 2013 Eine biograpische Skizze zur ersten Archäologin Sachsens. In: J.-E. Fries/D. Gutsmiedl-Schümann (Eds.), 
Ausgräberinnen, Forscherinnen, Pionierinnen. Ausgewählte Portraits früher Archäologinnen im Kontext ihrer Zeit (Münster 2013) 29-42.

KEGLER J.F. 2007 - J. F. Kegler, Das Azilien von Mas d'Azil. Der chronologische und kulturelle Kontext der Rückenspitzengruppen in Südwesteuropa. Thèse université de Cologne (Köln 2007).

KRAFT I. 2010 - Paläolithikum und Mesolithikum. In: R. Heynowski/R. Reiß (Eds.), Atlas zur Geschichte und Landeskunde von Sachsen. Beiheft zur Karte B I 1.1-1.5 Ur- und Frühgeschichte Sachsens. (Dresde 2010) 12-23.

KRAFT I. 2014 - I. Kraft, Die Entdeckung der Tiefenzeit, In: : S. Wolfram (Eds.), In die Tiefe der Zeit. 300.000 Jahre Menschheitsgeschichte in Sachsen. Das Buch zur Dauerausstellung, Dresde 2014, 24-30.

LINDENSCHMIT L. 1876 - Ueber die Thierzeichnungen auf Knochen der Thayinger Höhle. Archiv für Anthropologie 9 1876, 173-179.

MALLERY G. 1893 - Picture-writing of the American Indians. $10^{\text {th }}$ Annual Report of the Bureau of Ethnology of the Secretary of the Smithsonian Institution 1883-1889, 1893, 822-1290.

MÉLARD N., KRABATH S. 2005 - Die erste Archäologin Sachsens: auf den Spuren von Ida von Boxberg. Archæo 2, 2005, 33-37.
PÉQUART M., PÉQUART S.J. 1936 - De l'authenticité des galets colorés du Mas-d'Azil et de leur signification présumée. Congrès international d'anthropologie et d'archéologie préhistoriques, $12^{\mathrm{e}}$ Session, Toulouse-Foix 548-558.

PIETTE E. 1895 - Études d'ethnographie Préhistorique I. Réparation stratigraphique. L'Anthropologie 6, 1895, 275-292.

PIETTE E. 1896 - Études d'ethnographie Préhistorique III. - Les galets coloriés du Mas d'Azil. L'Anthropologie 7, 1896, 385-427.

PIETTE E. 1904 - Notions complémentiares sur l'Azilien. L'Anthropologie 14, 1903, 641-653.

SCHACHTMANN J. 2010 - Von der Prähistorischen Sammlung zum Landesmusum für Vorgeschichte: Die vorgeschichtliche Ausstellungen im Dresdner Wallpavillon. Archäologische Informationen 33, 2010, 59-68.

UNVERZAGT W., von JENNY W. 1935 - W. UnverzagtW. von Jenny, Zehn Jahre Museum für Vor- und Frühgeschichte 1924-1934. Berliner Museen 56, 1935, 2-15.

WELL 1926 - Scientific neglect of Mas D'Azil. Nature 118, 1926, 228.

WHITE 1992 - The history and research significance of the Logan Museum French Paleolithic collections. In: R. White, L. B. Breitborde (Hrsg.), French Paleolithic collections in the Logan Museum of Anthropology (Beloit 1992) 1-37. 
